\title{
Compressive Sensing Algorithm for Wireless Sensor Network Power Management
}

\author{
Jocelyn Flores Villaverde ${ }^{1}$, , Wen-Yaw Chung1*, Shih-Lun Chen ${ }^{1}$ \\ ${ }^{1}$ Chung Yuan Christina University, Zhongli, Taiwan R. O. C. \\ ${ }^{2}$ School of Electrical, Electronics and Computer Engineering, Mapua Institute of Technology, Manila, \\ Philippines.
}

* Corresponding authors. Email: eldanny@cycu.edu.tw

Manuscript submitted December 22, 2014; accepted April 27, 2015.

doi: 10.17706/ijcee.2015.7.3.199-205

\begin{abstract}
This paper presents lossless compression algorithm to minimize the power consumption of the wireless sensor network (WSN). The sensor and receiver node consumes more power when transmission of data is taking place. In addition, huge data are processed in the network which also consumes energy. The main contribution of this paper is to prolong the battery life of the sensors by using compressive sensing to lessen the transmission activity and data processing. CS is a lossless data compression technique that is used to represent the signals into sparse, linear encoding and transformation matrix, and sparse recovery. Discrete Fourier transform is used to get the nonzero coefficient of the signals. L1 minimization is used to recover the signal into its original form.
\end{abstract}

Key words: Compressive sensing, data compression, discrete fourier transform, L1 minimization, WSN.

\section{Introduction}

Data compression in wireless sensor network aims to reduce the power consumption of the system [1]. Wireless sensor network (WSN) is composed of different sensor nodes interconnected to each other and transmit the data to the base station. The sensor node measure physical conditions such as temperature, humidity, light intensity and carbon dioxide. It digitizes the inputs and transmits it to the base station which will be needed for monitoring and collecting the data. A typical sensor node is comprises of sensing unit or sensors, processing unit, communication unit and power supply unit. The sensing unit consists of analog to digital converter and the commercially available sensors. The processing unit is comprises of processor, memory and input-output ports. The communication unit is the radio transceiver and receiver of the WSN. Sensor nodes deployed in different areas need continuous power supply to meet its purpose. It process huge amount of data and this is one of the main factors that consume more energy and resources. Another factor that consumes more power in the activity of the WSN is data transmission. According to Kimura and Latifi [2] about $80 \%$ of the sensor node power is consumed in transmitting the data. Replacing the battery often may hinder accuracy and continuous monitoring of the system. Processing less data can contribute to extend the battery lifetime of the WSN.

WSN has a wide area of application. It is used to monitor health conditions of the patients such as heart rate, blood pressure, temperature and other physiological signals [3]-[5]. It is also applied in military applications as presented by Durisic et al. [6]. In addition, WSN is also installed in a greenhouse [7] to detect the soil moisture of the plant. Low power consumption is very important in WSN to continuously monitor 
the conditions of the signals. In this paper, a compressive sensing algorithm is used to minimize the data for reduction of transmission energy in the WSN. Different with the traditional method to sample all the signals with doubling frequency rate, the proposed method only sampled larger coefficients of the signals and disregarding the rest of the signals. It successfully reduced a huge part of power consumption in WSN. The rest of the paper is organized as follows. Section 2 discussed the background of compressive sensing algorithm. Section 3 discuss and simulate the compressive sensing algorithm. Section 4 presents the power consumption. Finally, Section 5 makes a brief conclusion of this work.

\section{Background of Compressive Sensing Algorithm}

Compressive sensing (CS) algorithm is a new paradigm in signal processing particularly for data acquisition [8], [9]. In a conventional sampling process known as the Shannon-Nyquist theorem [10], [11] the sampling rate must be twice as the highest frequency of the signal being measured. For instance, if the highest frequency of the signal is $30 \mathrm{KHz}$, the sampling rate must be greater than $60 \mathrm{KHz}$ in order to recover the original signal. In compressive sensing algorithm, it requires a sampling rate lower than the Nyquist rate to recover the original signal. It will only process the large coefficients and disregard the zero coefficients. This type of compression algorithm reduces the size of data sent and decreases the storage requirements of the system. Moreover, it will also lessen the transmission activity of the sensor node. Compressive sensing deals with acquiring the signal with a few numbers of measurements. It collects massive amount of data and compresses the data by neglecting most of the samples. Many researches in compressive sensing focus on image processing [12]-[14]. There are three parts in CS algorithm that need to be considered as illustrated in Fig. 1. The first part is the spare representation of the signal, second is the encoding and decoding of the measurement vectors. The last part is the recovery of the sparse signals.

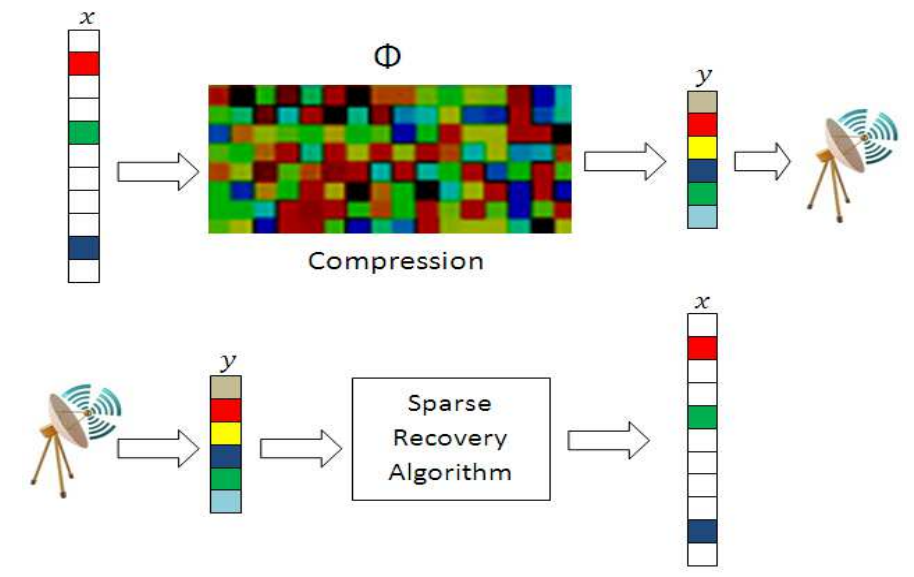

Fig. 1. Compressive sensing algorithm process.

The first consideration in a compressive sensing algorithm is the sparsity of the signal. In order to recover the signal in its original form, the signal should have a sparse representation. Suppose that $x \in \mathbb{R}^{N}$ is the sensor readings of $N$ signals and $\psi$ is a dictionary [15]. Assuming that the signals are sparse, the representation of the compressible signal can be expressed as:

$$
x=\sum_{i=1}^{N} s_{i} \psi_{i}
$$

where $x$ is the sparse representation of the signal and $\psi=\left\{\psi_{1}, \psi_{2}, \psi_{3} \ldots . \psi_{N}\right\}$ is the transformation basis, and $s$ is a $K$-sparse representation of $x$. A signal is said to be sparse if most of the coefficients are zero. Disregarding the zero coefficient will not loss much of the information of the signals. The compress signal 
can be represented in the form of equation 2 .

$$
y=\Phi x
$$

where the sensing matrix $\phi=\left\{\phi_{1}, \phi_{2}, \phi_{3}, \ldots \phi_{N}\right\}$ is a $M \times N$ CS matrix. The $y$ is now an $M \times 1$ measurement vector and $x$ is an $N \times 1$ column vector. Since $y$ is $M \times 1$ vector, therefore it is easier to transmit the data over the network.

Compressive sensing requires low coherent pairs. Coherence measures the largest correlation between any two elements. If the sensing vector $\phi$ and the sparsifying matrix $\psi$ are more incoherent, less number of samples is necessary in order to accurately recover the original signal [16]. The RIP can be connected to coherence property. The function and applicability of RIP in compressive sensing are presented in these works [17], [18]. It explained that RIP is suitable for to use as the sensing matrix property $\Phi$. This gives an accurate result for the under sampled measurements. RIP on the other hand is the solution to noise and imperfect measurements that may arise while acquiring the signals. "A sensing matrix is said to satisfy the RIP of order $K$ if there exist a constant $\delta \in(0,1)$ such that

$$
(1-\delta)\|x\|_{2}^{2} \leq\|\Phi x\|_{2}^{2} \leq(1+\delta)\|x\|_{2}^{2}
$$

where : $\delta K$ is the isometry constant and $x$ is the $n$ dimensional vectors [19]".

L1 minimization recovers the signal without knowing the number of nonzero coefficients in sparse representation. It is a very effective sparse recovery algorithm in a noisy measurement [20]-[22]. Replacing $l_{0}$ - norm by $l_{1}$ norm in linear programming is known as basis pursuit [23] is expressed as:

$$
\min _{x \in R^{N}}\|x\|_{1} \text { subject to } y=\Phi x
$$

\section{Simulation of Compressive Sensing Algorithm}

Data transmission consumes most of the energy in WSN. In order to save the energy, the reduction of data transmission over the network is the primary importance. The compressive sensing algorithm was simulated using MATLAB software to verify if the signal can be recovered to its original form. The sparsity of the signals are the non-zero coefficients. With this in mind the system will only focus on the large coefficients and zero coefficients will be neglected. Discrete Fourier transform was used to acquire the large coefficients of the signal. The sensing matrix $\phi=\left\{\phi_{1}, \phi_{2}, \phi_{3}, \ldots \phi_{N}\right\}$ is an $M \times N$ matrix, where $M$ « $N$. The sparse representation of the signals can be expressed using equation 1 . There are 512 samples with four different frequencies used in this simulation as shown in Fig. 2. After running the program, the 512 samples signal can be represented with only six large coefficients as depicted in Fig. 3. Using L1 minimization, it recovered the original signal using only the six large coefficients as illustrated in Fig. 4.

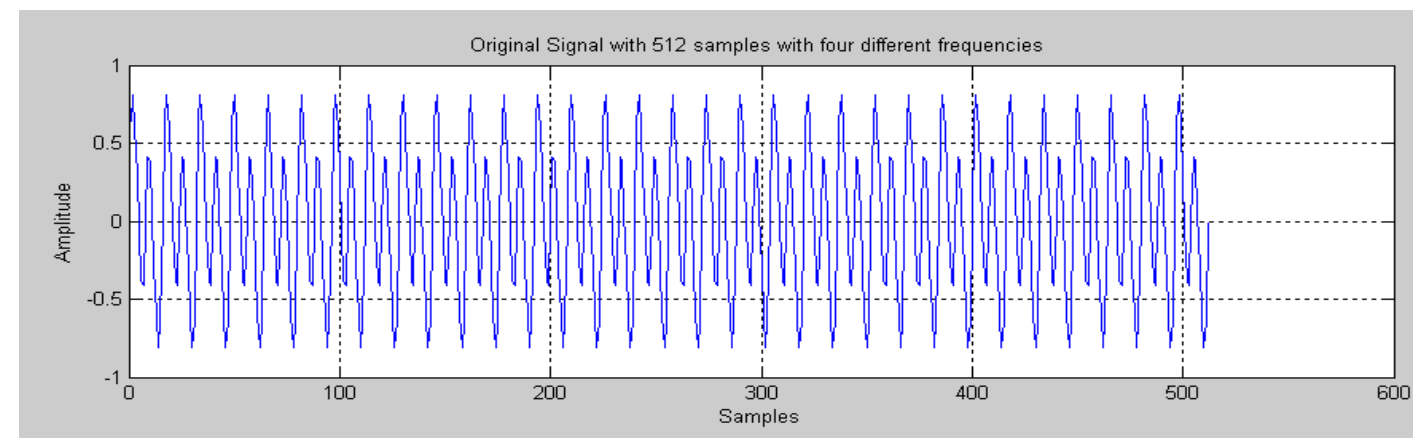

Fig. 2. Original signals with four different frequencies. 


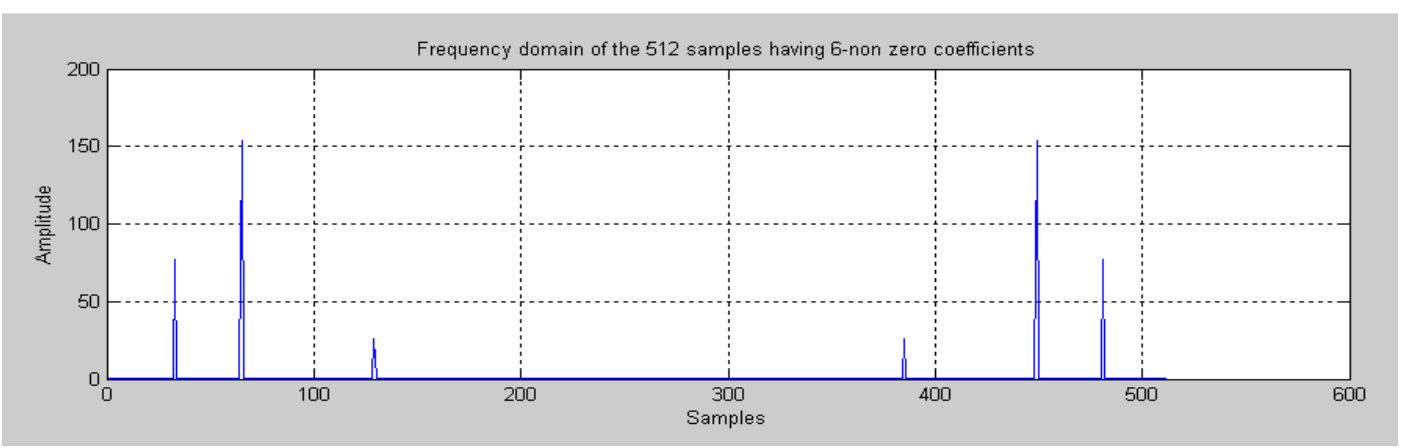

Fig. 3. 512 samples with 6 non-zero coefficients.

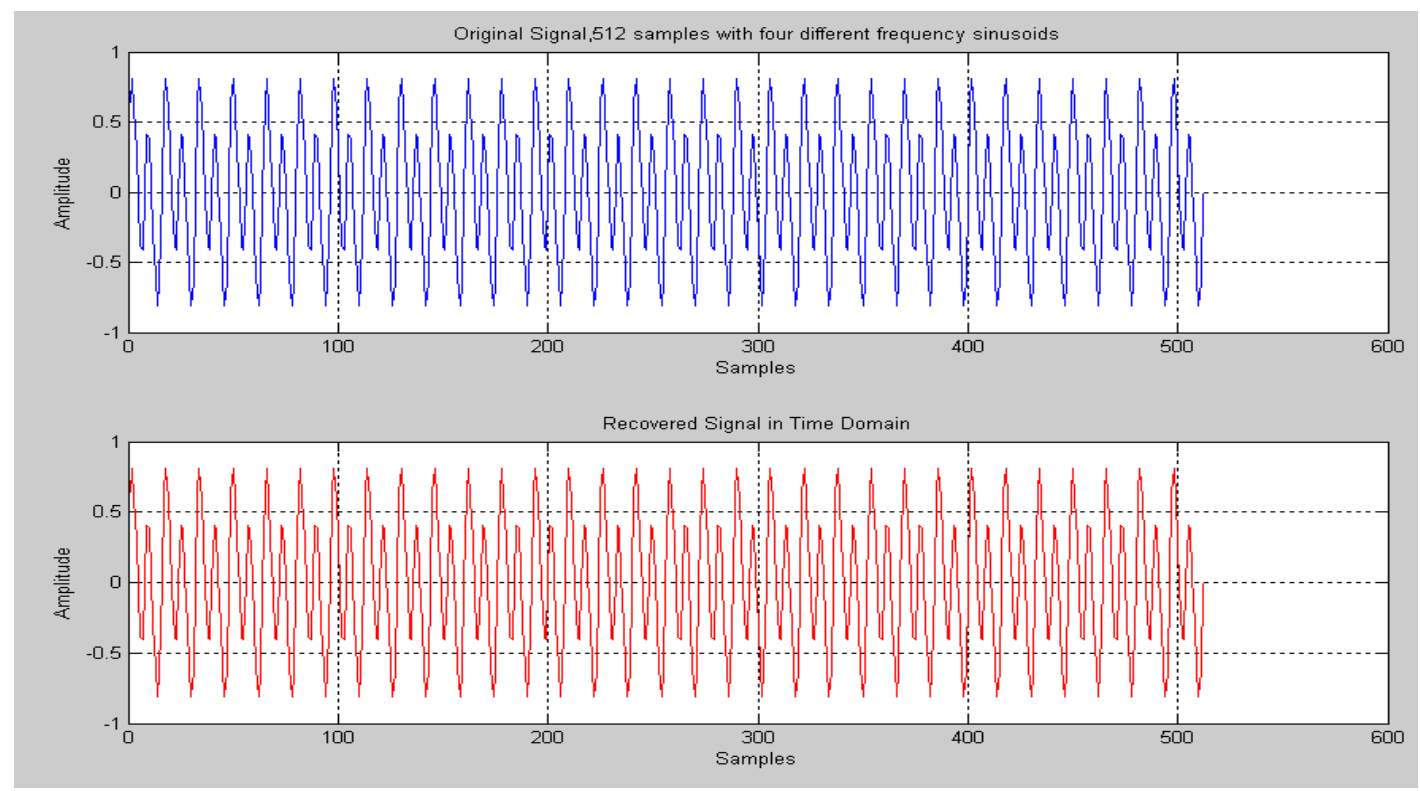

Fig. 4. Original signal compared to the recovered signal.

\section{Proposed Application of Compressive Sensing Algorithm}

In this paper, compressive sensing algorithm is proposed to implement in greenhouse monitoring system for power consumption. If the sensor node uses CC2530 based on the measurement setup in the Application Note of CC2530 [24], the current consumption of the microcontroller can be estimated using the operating mode of the microcontroller. For Operation 1, there is no sending of data to the receiver node. Therefore, the microcontroller will immediately go to sleep mode. For Operation 2, there is a message sending. Hence, the receiver will wait for the message and return an acknowledgement signal to the sending node. In Operation 3 , it is the same as Operation 1 but instead of transmitting the MAC data request it transmits the Toggle command. Operation 4 is the same as Operation 2. However, while waiting for the message to receive a Default Response, the command is sent. The current consumption of each mode is summarized in Table 1.

Table 1. Current Consumption of CC2530 [24]

\begin{tabular}{lcc}
\hline $\begin{array}{l}\text { Mode of } \\
\text { Operation }\end{array}$ & Time $(\mathrm{ms})$ & $\begin{array}{l}\text { Consumption } \\
\left(\mathrm{mA}^{*} \mathrm{mS}\right)\end{array}$ \\
\hline Operation 1 & 6.5825 & 100.5775 \\
Operation 2 & 23.865 & 388.4335 \\
Operation 3 & 7.2025 & 120.4175 \\
Operation 4 & 13.01 & 245.669 \\
\hline \hline
\end{tabular}


Assuming that the greenhouse monitoring system senses the temperature every 5 seconds and transmits the data 20 times within 24 hours. The total current consumption of the CC2530 can be computed as:

$$
\begin{gathered}
C C_{\text {total }}=C C_{\text {sleep }}+C C_{\text {op } 1} \times 17,260+C C_{\text {op } 3} \times 20+C C_{\text {op } 4} \times 20 \\
C C_{\text {ave }}=(86400+100.5775 \times 17260+120.4175 \times 20+245.669 \times 20) / 86400=21.18 \mathrm{uA}
\end{gathered}
$$

If the capacity of the battery is $2000 \mathrm{mAh}$, the battery life can be calculated as $2000 \mathrm{mAh} / 21.18 \mathrm{uA}=$ 94428.7 hours or equivalent to 3,934.5 days. Please refer to the application note [24] for the detailed discussion on the measurement setup of CC2530.

The average current consumption of the existing system under normal operation $\left(C C_{\text {normal }}\right)$ is $29.6 \mathrm{~mA}$, when the MAC return or sending data mode $\left(C C_{\text {sendstatus }}\right)$ is active the average current consumption of the system is $34.21 \mathrm{~mA}$. If MAC returns data every 60 seconds, the average current consumption of the existing greenhouse system is computed as:

$$
\begin{gathered}
60 \times 60 \times 24=86400 \mathrm{~s} \text { (per day) } \\
C C_{\text {ave }}=\left(C C_{\text {normal }}+C C_{\text {sendstatus }}\right) / 86400 \\
C C_{\text {ave }}=\frac{(29.6 \mathrm{~mA} \times 85260)+(34.21 \mathrm{~mA} \times 1140)}{86400}=29.66 \mathrm{~mA}
\end{gathered}
$$

The battery life $=2000 \mathrm{mAh} / 29.66 \mathrm{~mA}=67.43$ hours or equivalent to 2.8 days.

The proposed system will implement three modes of operation $C C_{\text {Sleep }}, C C_{\text {normal }}, C C_{\text {sendstatus. It will }}$ sense data every 60 seconds, transmit it every 300 seconds, and switch to Sleep mode for the remaining time within 24 hours. For the proposed system the average current consumption is computed as follows:

$$
\begin{gathered}
C C_{\text {ave }}=\left(C C_{\text {sleep }}+C C_{\text {normal }}+C C_{\text {sendstatus }}\right) / 86400 \\
C C_{\text {ave }}=\frac{(5 m A \times 84672)+(29.6 \mathrm{~mA} \times 1440)+(34.21 \mathrm{~mA} \times 288)}{86400}=5.5 \mathrm{~mA}
\end{gathered}
$$

The battery life $=2000 \mathrm{mAh} / 5.5 \mathrm{~mA}=363.63$ hours or equivalent to 15 days. Table 2 summarized the ideal average current consumption of the CC2530. It shows that using compressive sensing algorithm, the CC2530 will consume less power.

Table 2. Current Consumption

\begin{tabular}{lll}
\hline \hline Method & $\begin{array}{l}\text { Average Current } \\
\text { Consumption }\end{array}$ & $\begin{array}{l}\text { Battery } \\
\text { Life }\end{array}$ \\
\hline $\begin{array}{l}\text { Non compressive } \\
\text { sensing }\end{array}$ & $29.66 \mathrm{~mA}$ & 2.8 days \\
Compressive sensing & $5.5 \mathrm{~mA}$ & 15 days \\
\hline \hline
\end{tabular}

\section{Conclusion}

The compressive sensing algorithm reduced the amount of data that is being processed over the network. 
Limiting the number of measurements that are being processed will greatly reduce the power consumption of the system. In addition, the transmitting activity of the WSN is minimized. It prolonged the lifespan of the battery. Using fewer sampling measurements, the original signal can be recovered using L1 minimization. This can also be used to other WSN applications. For future work, the CS algorithm can be implemented in ASIC.

\section{Acknowledgment}

This work was supported by National Science Council, Taiwan (NSC), under Grant numbers of NSC 102-2221-E-033-063, MOST103-2218-E-033-004, MOST-103-2221-E-033-070, MOST-103-2622-E-033001-CC2 and MOST 103-2221-E-033-077.

\section{References}

[1] Srisooksai, T., Keamarungsi, K., Lamsrichan, P., \& Arak, A. (2012). Practical data compression in wireless sensor networks: A survey. Journal of Network and Computer Applications, 35(1), 37-59.

[2] Kimura, N., \& Latifi, S. (2005). A survey on data compression in wireless sensor networks. Proceedings of the International Conference on Information Technology: Coding and Computing: Vol. 2 (pp. 8-13).

[3] Ko, J. G., Lu, C., Srivastava, J., Terzis, A., \& Welsh, M. (2010). Wireless sensor networks for healthcare. Proceedings of the IEEE, 98(11), 1947-1960.

[4] Chen, C. A., Chen, S. L., Huang, H. Y., \& Luo, C. H. (2011). An asynchronous multi-sensor micro control unit for wireless body sensor networks (WBSNs). Sensors, 11, 7022-7036.

[5] Aminian, M., \& Naji, H. R. (2013). A hospital healthcare monitoring system using wireless sensor networks. Journal of Health and Medical Informatics, 2(2).

[6] Durisic, M., Tafa, Z., Dimić, G., \& Milutinović, V. (2012). A survey of military applications of wireless sensor networks. Proceedings of Mediterranean Conference on Embedded Computing (pp. 196-199).

[7] Chung, W.-Y., et al. (2013). Wireless sensor network based soil moisture monitoring system design. Proceedings of the 2013 Federated Conference on Computer Science and Information Systems (pp. 79-82).

[8] Donoho, D. L. (2006). Compressed Sensing. IEEE Trans. on Information Theory, 52, 1289-1306.

[9] Baraniuk, R. G. (2007). Compressive sensing. IEEE Signal Processing Magazine, 24, 118-121.

[10] Candes, E. J. \& Wakin, M. B. (2008). An introduction to compressive sampling. IEEE Signal Processing Magazine, 25, 21-30.

[11] Baranuik, R. G., Cevher, V., Duarte, M. F., \& Hegde, C. (2010). Model-based compressive sensing. IEEE Transactions on Information Theory, 56, 1982-2001.

[12] Robucci, R., Gray, J. D., Chui, L. K., Romberg, J., \& Haster, P. (2008). Compressive sensing on CMOS separable-transform image sensor. Proceedings of IEEE International Conference on Acoustics, Speech and Signal Processing (pp. 5125-5128). Las Vegas, USA.

[13] Arce, G. R., Brady, D. J., Carin, L., Arguello, H., \& Kittle, D. S. (2014). Compressive coded aperture spectral imaging. IEEE Signal Processing Magazine, 33, 105-115.

[14] He, J., Gao, M.-W., Zhang, L., \& Wu, H. (2013). Sparse signal recovery from fixed low-rank subspace via compressive measurement. Algorithm, 6, 871-882.

[15] Mallt, S., \& Zhang, Z. (1993). Matching pursuits with time-frequency dictionaries. IEEE Trans. on Signal Processing, 42, 3397-3415.

[16] Candes, E. J., \& Plan, Y. (2011). A probabilistic and RIPless theory of compressed sensing. IEEE Transactions on Information Theory, 57, 7235-7254.

[17] Nowak, J. H. R. (1997). A generalized restricted isometry property. University of Wisconsin Technical Report 1997, ECE-07-1. 
[18] Blanchard, J. D., Cartis, C., \& Tanner, J. (2011). Compressed sensing: How sharp is the restricted isometry property? SIAM Review, 53, 105-125.

[19] Wang, J., Kwon, S., \& Shim, B. (2012). Generalized orthogonal matching pursuit. IEEE Transactions on Signal Processing, 60, 6202-6216.

[20] Duarte-Carvajalino, J. M., \& Sapiro, G. (2009). Learning to sense sparse signals: Simultaneous sensing matrix and sparsifying dictionary optimization. IEEE Trans. on Image Processing, 18, 1395-1408.

[21] Zhang, Y. (2014). Theory of compressive sensing via L1-minimization: A non-RIP analysis and extensions. Form http://www.caam.rice.edu/ zhang/reports/tr0811.pdf

[22] Hugel, M., Rauhut, H., \& Strohmer, T. (2014). Remote sensing via L1-minimization. From http://rauhut.ins.uni-bonn.de/RadarCS.pdf

[23] Chen, S. S., Donoho, D. L., \& Saunders, M. A. (1998). Atomic decomposition by basis pursuit. SIAM J. Sci. Computing, 20, 33-61.

[24] Measuring power consumption of CC2530 with Z-stack. $\quad$ Form http://www.ti.com/lit/an/swra292/swra292.pdf

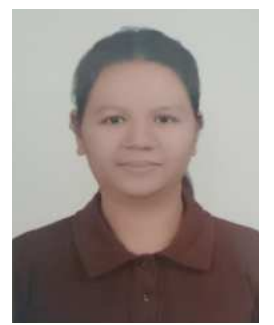

Jocelyn Flores Villaverde received her BS degree in computer engineering from the Technological Institute of the Philippines in 1997. She received her master degree in engineering with specialization in computer engineering from Pamantasan ng Lungsod ng, Maynila, Philippines in 2007. She is currently taking her PhD program in electronic engineering at Chung Yuan Christian University, Taiwan. She is a member of IAENG. Since 2005 she has been a faculty member in the Computer Engineering Department at Mapua Institute of Technology, Philippines. Her research interest includes digital circuit design, VLSI, data compression and wireless sensor network.

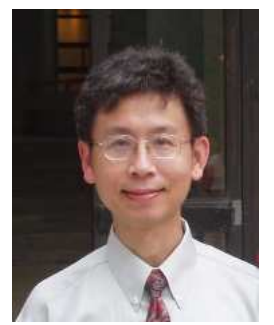

Wen-Yaw Chung received his BS, EE, and MS degrees from Chung Yuan Christian University, Chung Li, Taiwan in 1979 and 1981, respectively and a Ph.D. degree in electrical and computer engineering from Mississippi State University, USA in 1989. Subsequently, he joined the Advanced Microelectronics Division, Institute for Technology Development in Mississippi. In 1990 he worked as a design manager for the Communication Product Division, United Microelectronics Corporation, Hsin-Chu, Taiwan. Since 2006 he has been a full professor in the Department of Electronic Engineering and served as the director of the Electronics and Information Technology Center (EITC) at Chung Yuan Christian University. His research interests include mixed-signal VLSI design, biomedical IC applications, biosensor and actuator interfacing. He is a senior member of IEEE Circuits and Systems Society.

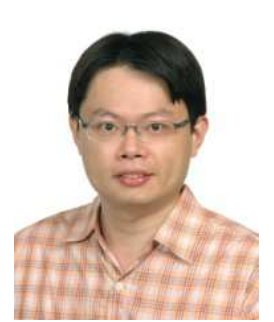

Shih-Lun Chen received the B.S. and M.S. degrees in electrical engineering from National Cheng Kung University, Tainan, Taiwan, in 2002 and 2004, respectively. He received the Ph.D. degree in the Department of Electrical Engineering, National Cheng Kung University Tainan, Taiwan in 2011. He was an assistant professor with the Department of Electronic Engineering, Chung Yuan Christian University in Taiwan, from 2011 to 2014. Since 2014, he has been an associate professor with the Department of Electronic Engineering, Chung Yuan Christian University in Taiwan. His current research interests include VLSI chip design, image processing, data compression, fuzzy logic control, wireless body sensor network, bio-medical signal processing, and reconfigurable architecture. 